\title{
Analysis of alpha wave epileptic EEG signals based on symbolic transfer
}

\author{
entropy \\ Xiang $\mathrm{Ye}^{1}$, Tian $\operatorname{Tian}^{1}$, Tianpei $\mathrm{Xu}{ }^{1}$, Jun Wang ${ }^{1, a}$ \\ ${ }^{1}$ Image Processing and Image Communications Key Lab., Nanjing Univ. of Posts \& Telecomm., \\ Nanjing, 210003, China \\ aemail: wangj@njupt. edu.cn
}

\section{Keywords: Symbolic transfer entropy; alpha wave; epileptic EEG;detection;prediction}

Abstract:

Epilepsy is a common neurological diseases caused by abnormal discharge of neurons in the brain. The attack is sudden and repeated .Therefore, advancing seizure prediction has important meaning for patients to take timely measures. In this paper,we can use the way of symbolic transfer entropy to study and analyze when epilepsy seizures. Through the EEG signal of epilepsy patients during attacked and normal human alpha wave is extracted, by using the method of symbolic transfer entropy for analysis and research. The study found that using this method can distinguish the normal EEG and EEG in patients with epilepsy. It was also found that the existence of nonlinear large amount of time series of EEG. This method also proved symbolic transfer entropy based on algorithm can be used to analyze the EEG signals fully and revealed the difference between epileptic EEG and normal EEG , which has clinical contribution to make certain detection and prediction of epilepsy.

\section{Introduction}

Epilepsy is commonly known as "epilepsy" or "epilepsy". Epilepsy is a kind of consciousness, feeling, nerve function and mental anomaly of plant disease that is caused by brain neurons abnormal discharge of paroxysmal[1-3]. There are many kinds of pathogenic factors. For symbolic transfer entropy algorithm[4]. We intend to firstly achieve a symbolic analysis of sequence, symbolic dynamics is the mathematical theory gradually developed in 20's 20th century. With the in-depth study of chaos, symbolic dynamics gradually become an important method for the analysis of chaos and complex sequence.

The analysis of symbolic time series[4,5] is developed as a new analysis method from the symbolic dynamic theory, chaotic time series and information theory. It can provide a fast, simple and efficient processing method for signal analysis. The symbolic essence is divided the continuous sign into several intervals according to certain rules, transfer the many continuous possible values into several symbolic sequences with discrete values. Although in this process, original sequence is missing some details, but dynamic characteristic is not lost. So it also can capture the large scale information and reduce the effects of dynamic noise and measurement noise. This process is also called "coarse-grained".

The purpose of this paper is applying the symbolic transfer entropy to the EEG signal for distinguishing epileptic EEG and normal EEG signal, which reveal the difference of epileptic EEG 
and normal EEG. It makes certain contribution to clinical detection and prediction of epilepsy.

\section{Principles}

The EEG and ECG signal are continuous time series. We usually symbolize the continuous time series $\mathrm{X}=\left\{x_{1}, x_{2}, \ldots, x_{i}, \ldots, x_{N}\right\}$. The length of the $\mathrm{X}$ is $\mathrm{N}$ and the symbolic series is $\mathrm{S}=\left\{s_{1}, s_{2}, \ldots, s_{i}, \ldots, s_{N}\right\}, s_{i} \in \mathrm{A},(\mathrm{A}=0,1,2,3)$. Symbolic methods used are as follows:

$$
S_{i}\left(x_{i}\right)= \begin{cases}0: & \mu_{1}<x_{i} \leq(1+a) \mu_{1} \text { or }(1+a) \mu_{2} \leq x_{i}<\mu_{2} \\ 1: & (1+a) \mu_{1}<x_{i} \leq \infty \text { or }-\infty \leq x_{i}<(1+a) \mu_{2} \\ 2: & (1-a) \mu_{1}<x_{i} \leq \mu_{1} \text { or } \mu_{2} \leq x_{i}<(1-a) \mu_{2} \\ 3: & (1-a) \mu_{2}<x_{i} \leq(1-a) \mu_{1}\end{cases}
$$

Here, $i=1,2, \cdots \cdots, N, \mu_{1}$ is the mean of $x_{i}$ in $\mathrm{X}$ sequence which is greater than zero, $\mu_{2}$ is the mean of $x_{i}$ in $\mathrm{X}$ sequence which is less than zero and a is Constant parameters, in the process of symbolization. If the a value is improper, The original time series will lose some details, so, we take $a=0.05$.

Transfer entropy is a parameter that quantifying the related degree of two systems or the degree of chaos. It can be used for physiological signal data processing of finite length.

The transfer of the definition of entropy is put forward by Schreiber in 2000.

$$
T_{J->}=\sum p\left(i_{n+1}, i_{n}^{(k)}, j_{n}^{(l)}\right) \log \frac{p\left(i_{n+1} \mid i_{n}^{(k)}, j_{n}^{(l)}\right)}{p\left(i_{n+1} \mid i_{n}^{(k)}\right)}
$$

$i_{n}, j_{n}$ represent the state at $\mathrm{n}$ times of tow sequences: I sequence, $\mathrm{J}$ sequence. $i_{n}^{(k)}$ represents a string of length $\mathrm{k}$ as $i_{n-k+1}, \cdots \cdots, i_{n}$, By the same token, $j_{n}^{(l)}$ represents a string of length 1 as $j_{n-k+1}, \cdots \cdots, j_{n}$. Usually in order to simplify calculation, we generally take $\mathrm{k}=1, \mathrm{I}=1$.

For symbolic transfer entropy algorithm, the realization of the method is as follows: a time sequence $x(t), t=1,2, \ldots$ then the phase space reconstruction for:

$$
X_{t}=\{x(t), x(t+\tau), \cdots \cdots, x(t+(m-1) \tau)\}
$$

Any arbitrary phase point have $m$ dimensional components, where $m$ represents embedding dimension, $\tau$ represent delay time. Then we put the $m$ dimensional components of $X_{t}$ in ascending order:

$$
X_{t}=\left\{x\left(t+\left(k_{t 1}-1\right) \tau\right), x\left(t+\left(k_{t 2}-1\right) \tau\right), \cdots \cdots, x\left(t+\left(k_{t m}-1\right) \tau\right)\right\}
$$

It can take the $k_{t 1}, k_{t 2}, \cdots \cdots, k_{t m}$ to construct a new time series, $x_{t}=\left(k_{t 1}, k_{t 2}, \cdots \cdots, k_{t m}\right)$. 
In this agreement: if two elements have equal value, $x\left(t+\left(k_{t 1}-1\right) \tau\right)=x\left(t+\left(k_{t 2}-1\right) \tau\right)$, we take $x\left(t+\left(k_{t 1}-1\right) \tau\right) \leq x\left(t+\left(k_{t 2}-1\right) \tau\right)$ and we think that $X_{t}$ have $m$ ! values.

The same time on the same individuals collected two lead EEG time sequence. Two time sequence number $\left\{x_{i}\right\}$ and $\left\{y_{i}\right\}$, separately for each of the whole sequence may appear in the statistics. The relative frequency and calculate the various situation appears as a probability. Calculating the STE, the calculation process of STE are as follows:

$$
\begin{gathered}
h_{1}=-\sum p\left(x_{t+1}, x_{t}, y_{t}\right) \log _{2} p\left(x_{t+1} \mid x_{t}, y_{t}\right) \\
h_{2}=-\sum p\left(x_{t+1}, x_{t}, y_{t}\right) \log _{2} p\left(x_{t+1} \mid x_{t}\right) \\
T_{Y, X}^{S}=h_{2}-h_{1}=\sum p\left(x_{t+1}, x_{t}, y_{t}\right) \log _{2} \frac{p\left(x_{t+1} \mid x_{t}, y_{t}\right)}{p\left(x_{t+1} \mid x_{t}\right)} \\
=\sum p\left(x_{t+1}, x_{t}, y_{t}\right) \log _{2} \frac{p\left(x_{t+1}, x_{t}, y_{t}\right) p\left(x_{t}\right)}{p\left(x_{t}, y_{t}\right) p\left(x_{t}, x_{t+1}\right)}
\end{gathered}
$$

In the formula, $h_{1}$ express $y_{t}$ and $x_{t+1}$ are not independent by $x_{t}, y_{t}$ to test the entropy of next sampling point $x_{t+1}$. The formula $h_{2}$ expresses $y_{t}$ and $x_{t+1}$ is independent by $x_{t}$ to test the entropy of next sampling point which describing the degree of correlation and randomness between two time sequence quantitatively.

\section{Data processing and analysis}

In this paper, by the use of data provided by General Hospital of Nanjing Military Region collected from clinical diagnosis information, we take the EEG signal from epilepsy patients and normal people in the database. Epilepsy patients and normal subjects were divided into two groups and each group had ten objects. The EEG data for each object includes 24 lead. The record length is greater than 1 minutes and the sampling period is $1024 \mathrm{~Hz}$. Two groups include both male and female. We study the transfer entropy of two column lead data:Fp1 and Fp2 (experiment proof, the symbolic transfer entropy of Fp1 and Fp2 have good discrimination. So we took Fp1,Fp2 lead as the object of study). In the following, we recorded epilepsy group "epilepsy", normal group for "normal".

By the image we can find, Alpha wave extracted regularly, and filtering algorithm is more reasonable. Epileptic EEG frequency diagram of alpha wave was in lead Fp1 and the single mainly concentrated in the $8 \sim 13 \mathrm{~Hz}$, which meeting the requirements. In the experiment, we take 1000 , 2000、3000 ... 8000 points, to calculate the transfer entropy as shown in Figure 1. 

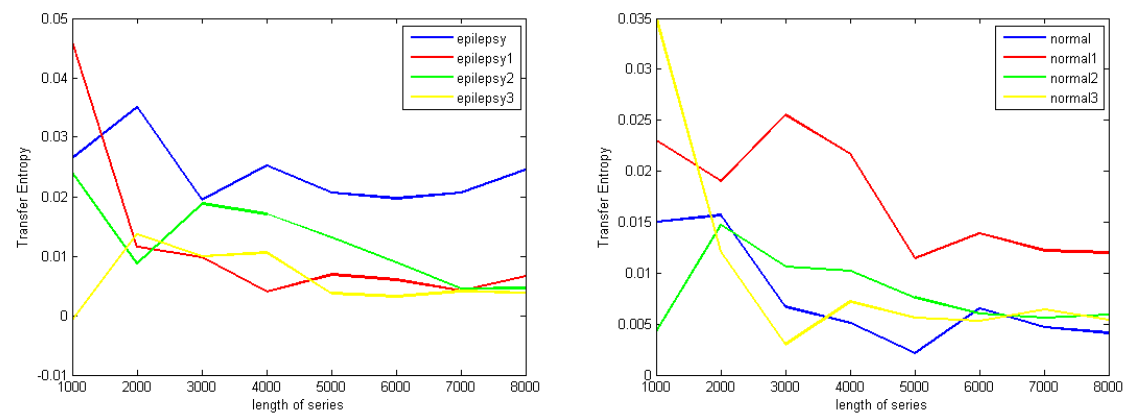

Figure 1 the epileptic and normal EEG entropy transfer value in different lengths.

The results showed that, the value of transfer entropy in each sequence is almost convergent. After reaching a certain length., thousands of points can get consistent results. So considering the epileptic EEG and normal EEG pattern, we go to select the $L=6000$ as the selection of length. This symbolic transfer entropy method needs less data with high computational efficiency.

For epileptic EEG and normal EEG, we are beginning to take a length of 6000 data from the initial moment. To calculate the transfer entropy, we take a length of 6000 data start to the 1000 point. Calculating transfer entropy and obtaining two kind of signal transfer entropy change with time is shown in Fig. 2.

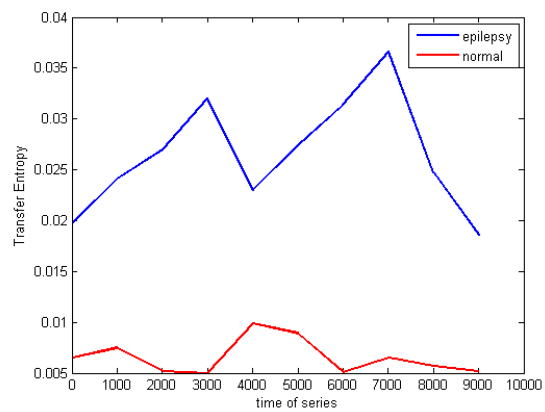

Figure 2 different periods the transfer entropy value of epileptic EEG.

Finally, In order to verify whether there is a significant difference between the second and third step experiment calculated signal transfer entropy, we have them independent samples $t$ test.

We use SPSS software for two independent samples T test signal. $t=6.409>t_{0.05(8)}=1.8595$. So we consider that the length of the analysis and comparison of methods can clearly distinguish epileptic EEG and normal EEG.

\section{Conclusions}

Epilepsy is a brain neurons disease caused by excessive discharge of brain dysfunction. The onset of brain damage is irreversible. Therefore, seriously endanger human health. In this paper, we can use the way of symbolic transfer entropy to study and analyze when epilepsy seizures. Fully proved symbolic transfer entropy-based algorithm can be used to analyze EEG. That revealed the difference between epilepsy EEG and normal EEG. We can make some clinical contribution for epilepsy detection and prediction. 


\section{Acknowledgements}

In this paper, the project supported by the National Natural Science Foundation of China (Grant Nos. 61271082,61201029,61102094,61401518), the Natural Science Foundation of Jiangsu Province (Grant Nos.BK20141432), the Foundation of Nanjing General Hospital of Nanjing Military Command (2014019) and the Fundamental Research Funds for the Central Universities (FY2014LX0039).

\section{Reference}

[1] Meng Q F, zhou W, Chen Y H, Peng Y H 2010 Acta Phys. Sin. 59 123-130

[2] Ma Q L, Bian C H, Wang J 2010 Acta Phys. Sin. 59 4480-4484

[3] Wang J, Ma Q-L 2008 Chin. Phys. B 174424

[4] Staniek M, Lehnertz K 2007 Phys. Rev. Lett. 99204101

[5] Shen W, Wang J 2010 Acta Phys. Sin. 60118702. 